\section{Aislamiento de Staphylococcus aureus hetero-resistente a vancomicina en Hospital Clínico Regional de Concepción, Chile}

Félix Vega, Pedro Alarcón, Mariana Domínguez, Helia Bello, Gisela Riedel, Sergio Mella, Alejandro Aguayo y Gerardo González-Rocha

Isolation of Staphylococcus aureus hetero-resistant to vancomycin (hVISA) in the Regional Hospital of Concepción, Chile

Methicillin-resistant Staphylococcus aureus (MRSA) is widely distributed in hospital environments, causing serious infections, mainly the bloodstream, surgical site infection and pneumonia. Vancomycin (VAN) is the antibiotic of choice for treating severe MRSA infections; however, nowadays worldwide resistant strains (VRSA), with intermediate susceptibility (VISA) and decreased susceptibility or hetero-resistance to VAN (hVISA) have been reported, related to treatment failure and increased mortality. This report describes the first confirmed isolation of MRSA with hVISA phenotype in a public hospital in Chile. Chile.

Key words: Staphylococcus aureus, MRSA, vancomycin, hVISA,

Palabras clave: Staphylococcus aureus, SARM, vancomicina, SAIVh, Chile.

$S$ taphylococcus aureus resistente a meticilina (SARM) se encuentra ampliamente distribuido en ambientes hospitalarios, provocando infecciones graves, principalmente del torrente sanguíneo, infección de sitio quirúrgico y neumonía ${ }^{1}$. En Chile, el Grupo Colaborativo de Resistencia Antimicrobiana informó, para el año 2012, 51,7\% de susceptibilidad a cloxacilina en pacientes adultos hospitalizados y $84,9 \%$ en niños ${ }^{2}$. La resistencia a meticilina se encuentra codificada en los genes mec, insertos en el cassette cromosomal denominado SCCmec. Se han descrito 11 tipos y varios subtipos, de los cuales el tipo I, II y III predominan en cepas de origen hospitalario ${ }^{3}$. Vancomicina (VAN) es el antimicrobiano de elección para tratar infecciones graves por SARM; sin embargo, en la actualidad se ha informado cepas resistentes (SARV), con susceptibilidad intermedia (SAIV) y susceptibilidad disminuida o con hetero-resistencia (SAIVh) a

\footnotetext{
Universidad de Concepción, Concepción, Chile.

Facultad de Ciencias Biológicas, Departamento de Microbiología.

Laboratorio de Investigación en Agentes Antibacterianos (FV, MD, HB, GG).

Facultad de Medicina, Departamento de Medicina Interna (GR, SM, AA).

Instituto de Salud Pública, Santiago, Chile (PA).
}

Declaración de conflicto de interés: Sergio Mella ha recibido honorarios como conferencista de Laboratorios Pfizer y MSD. Los otros autores no tienen conflictos de interés.

Fuente de financiamiento parcial por Novartis Chile S.A.

Recibido: 30 de abril de 2015 / Aceptado: 28 de julio de 2015

Correspondencia a:

Gerardo González-Rocha

ggonzal@udec.cl
VAN, relacionadas a falla en el tratamiento y aumento de la mortalidad ${ }^{4}$. Los mecanismos moleculares que originan estos fenotipos de resistencia son específicos. En el caso de SARV se produce por adquisición del gen vanA desde cepas de Enterococcus spp. lo que produce elevados niveles de resistencia (concentración inhibitoria mínima, CIM de VAN $\geq 16 \mu \mathrm{g} / \mathrm{mL}$ ), a diferencia de SAIV y SAIVh, donde se altera la estructura de la pared bacteriana impidiendo el acceso del antimicrobiano a su sitio de acción, generando una elevación leve de los niveles de CIM de VAN $(4-8 \mu \mathrm{g} / \mathrm{mL}$ para SAIV y $<$ $2 \mu \mathrm{g} / \mathrm{mL} \mathrm{SAIVh})^{4}$. Hasta el año 2012 se habían notificado 12 casos de SARV, principalmente en los E.U.A., causando infecciones de piel y tejidos blandos en pacientes con enfermedades crónicas que se encontraban colonizados con Enterococcus faecalis portador del gen vanA. Posteriormente, el año 2013 se reportó el primer aislado en Latinoamérica de SARV de un paciente con características similares ${ }^{5}$. La primera cepa con fenotipo SAIV de $S$. aureus fue reportada en Japón el año 1997 y se denominó Mu50, con una CIM de $8 \mu \mathrm{g} / \mathrm{mL}$ a VAN y fue aislada de una herida operatoria ${ }^{6}$. Sin embargo, en 1996 se había aislado la cepa Mu3, la primera con fenotipo SAIVh, desde un paciente con neumonía por SARM tratada 12 días con VAN, y que se postula sería el origen de Mu50 $0^{7,8}$. Posteriormente, surgen reportes en todo el mundo incluso en análisis retrospectivos con cepas almacenadas desde $1987^{4}$. Dentro de los factores asociados al incremento de la CIM a VAN en el caso de cepas SAMR y SAIVh se encuentran el tratamiento previo con este glicopéptido y la estancia en unidades de cuidados intensivos 9 .

En Chile no se han detectado cepas resistentes a VAN entre los aislados de $S$. aureus y la distribución de las CIM es bastante homogénea, con valores inferiores a $1,0 \mu \mathrm{g} / \mathrm{mL}$, manteniéndose dentro del rango de susceptibilidad de acuerdo a los puntos de corte recomendados por CLSI ${ }^{10}$.

En esta comunicación se describe el primer hallazgo de una cepa de $S$. aureus SAIVh en Chile, aislada en una mujer de 62 años internada en el Hospital Clínico Regional de Concepción con antecedentes de una diabetes mellitus tipo 2, hipertensión arterial y enfermedad renal crónica secundaria a nefropatía diabética en hemodiálisis. Había recibido tratamiento con vancomicina previamente por una osteomielitis secundaria a un pie diabético por S. epidermidis resistente a meticilina y por dos episodios de bacteriemias por SARM asociadas a CVC de hemodiálisis.

El estudio de susceptibilidad de la cepa de $S$. aureus, por método de difusión en agar, mostró resistencia a cefoxitina, eritromicina y clindamicina, sugiriendo resistencia a meticilina y de tipo MLS $_{B}$ constitutiva, respectivamente. En cambio, fue susceptible a rifampicina, sulfametoxazol-trimetoprim, tetraciclina y linezolid (LZD); concordante con el predominio del clon chileno-cordobés en nuestro país. Se determinó la CIM por método de dilución seriada en agar y epsilometría a VAN y por microdilución en caldo a daptomicina (DAP) y LZD. Los resultados indicaron susceptibilidad a VAN (CIM 2,0 $\mu \mathrm{g} / \mathrm{mL}$ ) y LZD (CIM 1,0 $\mu \mathrm{g} / \mathrm{mL}$ ), pero no susceptible a DAP (CIM 2,0 $\mu \mathrm{g} / \mathrm{mL})$. La cepa presentó el gen mecA, el SCCmec tipo I y fue negativa para el gen $p v l$, mediante amplificación por reacción de polimerasa en cadena (RPC). La subtificación de esta cepa por MLST (http://saureus.mlst.net/) determinó que se trata del secuencio-tipo ST267, que es un ST no descrito con anterioridad y que pertenece al complejo clonal CC5. La confirmación que esta cepa corresponde a $S$. aureus SAIVh se realizó en el Instituto de Salud Pública mediante el método de glycopeptide resistance detection $(\mathrm{GRD})^{11}$ (Figura 1).

La prevalencia de SAIVh es difícil de determinar por el amplio número de metodologías para su detección, pero se estima que corresponde a 1,3\% de los aislados de SARM ${ }^{12}$. En Latinoamérica existe escasa información 


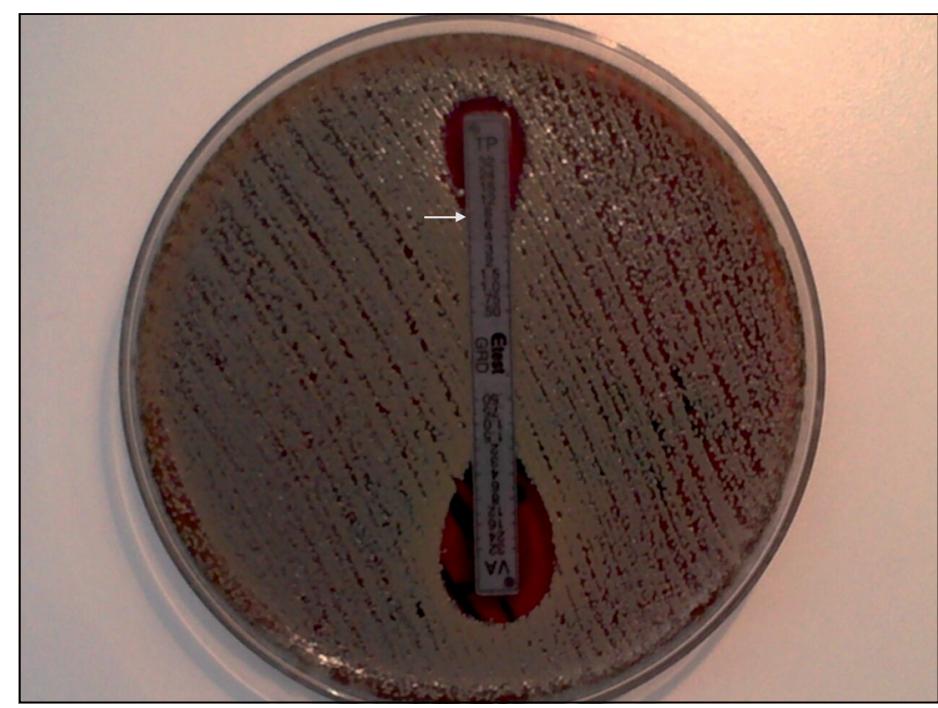

Figura 1. Confirmación de SAIVh por método de Glycopeptide Resistance Detection (GRD e-test). Se observa una CIM de $8 \mu \mathrm{g} / \mathrm{mL}$ (flecha) para teicoplanina (TP), lo que confirma el fenotipo SAIVh para la cepa estudiada. VA: vancomicina.

de aislados de SAIVh; sin embargo, recientemente Di Gregorio y cols. ${ }^{13}$, comunicaron una prevalencia de 3,3\% de SAIVh en un hospital universitario de Argentina.

La susceptibilidad intermedia y la hetero-resistencia a VAN se produce por una serie de cambios estructurales de la pared celular como reducción de la expresión de proteínas de unión a penicilina (PBP) y disminución de entrecruzamientos que dejan libres residuos D-ala D-ala capaces de unir VAN impidiendo que ésta llegue a su sitio de acción ${ }^{14}$. Diversas mutaciones en un pequeño número de genes reguladores como walKR, graRS, vraSR, que corresponden a sistemas de quorum sensing de dos componentes, y el gen $r p o B$, que codifica para la subunidad $B$ de la ARN polimerasa, han sido asociadas a los fenotipos SAIV y SAIVh ${ }^{15}$.

Dentro de las metodologías para la detección de SAIVh se encuentra el análisis de perfiles poblacionales, que corresponde al estándar de oro; sin embargo, es una metodología laboriosa y que consume tiempo por lo que no se puede realizar de rutina. Por esta razón, se han desarrollado otras técnicas como el macrométodo E-test y la GRD E-test que consiste en inocular una suspensión 0,5 McFarland en una placa de Mueller Hinton adicionada con $5 \%$ de sangre cordero e incubar por $48 \mathrm{~h}$ a $35^{\circ} \mathrm{C}$, incorporando una tira de E-test GRD $^{11}$, la cual presenta doble gradiente de antibacteriano, por un extremo VAN y por el otro teicoplanina. Si posterior a la incubación se observa una CIM $\geq 8 \mu \mathrm{g} / \mathrm{mL}$, a cualquiera de los dos glicopéptidos, se considera que la cepa corresponde al fenotipo SAIVh ${ }^{16}$.

Hasta donde tenemos conocimiento, y no obstante existe un informe de cepas de $S$. aureus inhibidas con $8 \mu \mathrm{g} / \mathrm{mL}$ de $\mathrm{VAN}^{17}$, este trabajo constituye el primer reporte confirmado de una cepa de $S$. aureus SAIVh en Chile. Es fundamental mantener programas de vigilancia que permitan detectar la presencia de cepas con este fenotipo en pacientes con factores de riesgo como tratamiento prolongado con VAN, de modo de predecir fracaso con este fármaco y optimizar la terapia antimicrobiana.

Agradecimientos. Agradecemos a Novartis Chile S.A. por financiar parte de esta investigación y al Laboratorio Clínico del Hospital "Dr. Guillermo Grant Benavente", de Concepción (Chile), por colaborar en la recolección de las cepas incluidas en este estudio.

\section{Resumen}

Staphylococcus aureus resistente a meticilina (SARM) se encuentra ampliamente distribuido en ambientes hospitalarios, provocando infecciones graves, principalmente del torrente sanguíneo, infección de sitio quirúrgico y neumonía. Vancomicina (VAN) es el antimicrobiano de elección para tratar infecciones graves por SARM; sin embargo, actualmente en el mundo se han comunicado cepas resistentes (SARV), con susceptibilidad intermedia (SAIV) y susceptibilidad disminuida o con hetero-resistencia a VAN (SAIVh), relacionadas a falla terapéutica y aumento de la mortalidad. Describimos el primer aislado de SARM con fenotipo SAIVh en un hospital público de Chile.

\section{Referencias bibliográficas}

1.- Rice L B. Antimicrobial resistance in Gram-positive bacteria. Am J Infect Control 2006; 34 (5 Suppl 1): vS11-9.

2.- Cifuentes M, Silva F, García P, Bello H, Briceño I, Calvo M, et al. Susceptibilidad antimicrobiana en Chile 2012. Rev Chilena Infectol 2014; 31: $123-30$.

3.- Shore A C, Coleman D C. Staphylococcal cassette chromosome mec: recent advances and new insights. Int J Med Microbiol 2013; 303: 350-9.

4.- Howden B P, Davies J K, Johnson P D, Stinear T P, Grayson M L. Reduced vancomycin susceptibility in Staphylococcus aureus, including vancomycinintermediate and heterogeneous vancomycin-intermediate strains: resistance mechanisms, laboratory detection, and clinical implications. Clin Microbiol Rev 2010; 23: 99-139.

5.- OPS. Alerta epidemiológica: Staphylococcus aureus resistente a vancomicina. Disponible: http://www.paho.org/hq/index.php?option=com docman\&task=doc_view\&gid=22189\&Itemid $=($ fecha de acceso: 20 de julio de 2015).

6.- Hiramatsu K, Hanaki H, Ino T, Yabuta K, Oguri T, Tenover F C. Methicillinresistant Staphylococcus aureus clinical strain with reduced vancomycin susceptibility. J Antimicrob Chemother 1997; 40: 135-6.

7.- Hiramatsu K, Aritaka N, Hanaki H, Kawasaki S, Hosoda Y, Hori S, et al. Dissemination in Japanese hospitals of strains of Staphylococcus aureus heterogeneously resistant to vancomycin. Lancet 1997; 6: 1670-3.

8.- Rodríguez C A, Vesga O. Staphylococcus aureus resistente a vancomicina. Biomédica 2005; 25: 575-87.

9.- Lodise T P, Miller C D, Graves J, Evans A, Graffunder E, Helmecke M, et al. Predictors of high vancomycin MIC values among patients with methicillinresistant Staphylococcus aureus bacteremia. J Antimicrob Chemother 2008; 62: 1138-41.

10.- CLSI. Performance Standards for Antimicrobial Susceptibility Testing; Twenty-Fifth Informational Supplement. CLSI document M100-S25. Wayne, PA: Clinical and Laboratory Standards Institute; 2015.

11.- Yusof A, Engelhardt A, Karlsson A, Bylund L, Vidh P, Mills K, et al. Evaluation of a new E-test vancomycin-teicoplanin strip for detection of glycopeptide-intermediate Staphylococcus aureus (GISA), in particular, heterogeneous GISA. J Clin Microbiol 2008; 46: 3042-7.

12.- van Hal S J, Paterson D L. Systematic review and meta-analysis of the significance of heterogeneous vancomycin-intermediate Staphylococcus aureus isolates. Antimicrob Agents Chemother 2011; 55: 405-10.

13.- Di Gregorio S, Perazzi B, Ordoñez A M, De Gregorio S, Foccoli M, Lasala M B, et al. Clinical, microbiological, and genetic characteristics of 
heteroresistant vancomycin-intermediate Staphylococcus aureus bacteremia in a teaching hospital. Microb Drugs Resist 2015; 21: 25-34.

14.- Fridkin S K. Vancomycin-intermediate and-resistant Staphylococcus aureus: what the infectious disease specialist needs to know. Clin Infect Dis 2001; 32: $108-15$.

15.- Howden B P, Peleg A Y, Stinear T P. The evolution of vancomycin intermediate Staphylococcus aureus (VISA) and heterogenous-VISA. Infect Genet Evol 2014; 21: 575-82.
16.- van Hal S J, Wehrhahn M C, Barbagiannakos T, Mercer J, Chen D, Paterson $\mathrm{D}$ L, et al. Performance of various testing methodologies for detection of heteroresistant vancomycin-intermediate Staphylococcus aureus in bloodstream isolates. J Clin Microbiol 2011; 49: 1489-94.

17.- Otth L, Wilson M, Bustamante N, Fernández H, Otth C. Susceptibilidad antimicrobiana y patrones de resistencia de Staphylococcus aureus aislados de pacientes y portadores en la ciudad de Valdivia. Rev Chilena Infectol 2008; 25: 175-8. 